\title{
Lack of effort or lack of ability? Robot failures and human perception of agency and responsibility
}

\author{
Sophie van der Woerdt ${ }^{\mathrm{a}}$ Willem F.G. Haselager ${ }^{\mathrm{b}}$ \\ ${ }^{\mathrm{a}}$ Dpt. of Psychology, ${ }^{\mathrm{b}}$ Dpt. of Artificial Intelligence, Radboud University, \\ Comeniuslaan 4, $6525 \mathrm{HP}$, Nijmegen
}

\begin{abstract}
Research on human interaction has shown that attributing agency to another agent has substantial consequences for the way we perceive and evaluate its actions. Specifically, considering an agent's actions related to either effort or ability can have important consequences for the attribution of responsibility. This study indicates that participants' interpretation of a robot failure in terms of effort -as opposed to abilitysignificantly increases their attribution of agency and -to some extent- moral responsibility to the robot. However a robot displaying lack of effort does not lead to the level of affective and behavioural reactions of participants normally found in reactions to other human agents.
\end{abstract}

\section{Introduction}

Currently, much debate is devoted to the question of how we should deal with harm caused by robots (Asaro 2013; Singer 2011). Research on anthropomorphism (Duffy 2003; Złotowski, Strasser \& Bartneck 2014), blame (Moon \& Nass 1998; Serenko 2007; Kim \& Hinds 2006; You, Nie, Suh \& Sundar 2011; Koay, Syrdal, Walters \& Dautenhahn 2009; Vilaza, Haselager, Campos, \& Vuurpijl 2014; Malle, Scheutz, Arnold, Voiklis, \& Cusimano 2015; Malle, Scheutz, Forlizzi, \& Voiklis 2016) and examples of media and pop culture speaking of 'robot laws' (Clarke 1994) underline the possibility of humans - perhaps inappropriately-attributing moral responsibility to automated systems. Although legal solutions have been proposed for dealing with such conflicts (Asaro 2013), in daily life this may still have undesired implications. Owners and developers of robots may (unknowingly) distance themselves from potential harms caused by their robots (Coleman 2004), causing responsibility to become diffused. There- fore, it is relevant to find out what factors contribute to the attribution of agency and responsibility in robots.

Extensive work on attributional processes in human interaction reveals that the perception of an agent's effort and abilities are central determinants in the attribution of agency and moral responsibility (Weiner 1995). This, in turn, is strongly related to fundamental affective and behavioural reactions such as sympathy, rejection, altruism and aggression (Weiner 1995; Rudolph, Roesch, Greitemeyer, \& Weiner 2004). Yet, with regard to human robot interaction (HRI), little is known about the attribution of agency and moral responsibility.

In this study, we applied Weiner's Theory of Social Conduct (Weiner 1995) to HRI by showing participants videos of robots (Aldebaran's NAO) failing tasks in ways that could be interpreted as due to either lack of ability (LA-condition; e.g. dropping an object) or lack of effort (LE-condition; e.g. throwing away an object, fig. 1). We expected that a display of lack of effort would incite the illusion of a robot having agency over its actions. In addition, we expected that a robot's lack of effort would have little effect on the attribution of moral responsibility to the robot, compared to a display of lack of ability.

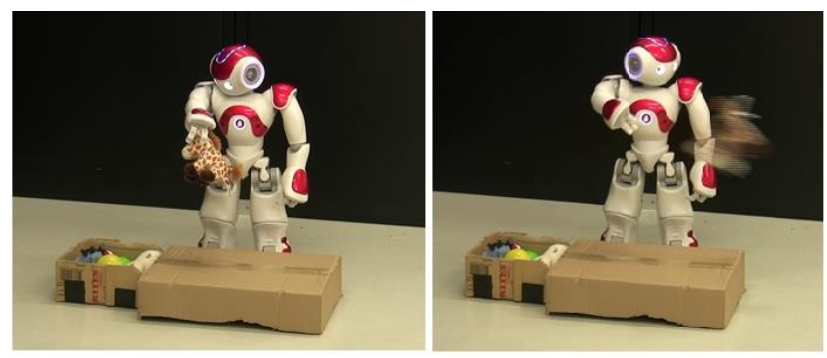

Fig. 1: sample frames of (a) a robot displaying the intention of putting a toy in a box, (b) the robot throwing the toy away instead (LE-condition). 


\section{Method}

In an online survey, sixty-three participants $\left(M_{\text {Age }}=25,5\right.$, $S D=9,7$; drawn from a university population) were shown a video of about 30-60 seconds portraying a situation in which a NAO robot was shown failing a task either due to lack of ability or lack of effort. Seven of such scenarios were presented ${ }^{1}$. After each video, participants were asked to fill in a questionnaire containing scales of agency (five questions about the robot's control over the situation and its ability to make its own decisions), and responsibility (twelve questions on attributed blame and kindness, affective and behavioural reactions). Additionally, scales were included measuring the participant's estimate of the robot's experience (e.g. having beliefs, desires, intentions, emotions), predictability, propensity to do damage, trustworthiness and nonanthropomorphic features (e.g. strength, efficiency, usefulness) ${ }^{2}$.

For analysis, mean scores of each scale (range 1-5) were calculated and transposed to Z-scores. Since reliability and goodness-of-fit for the scale of responsibility was questionable, items of this scale were analyzed separately. In order to answer our main questions, a GLM multivariate analysis was performed with the composite means of agency, experience, predictability, propensity to do damage, and each item related to responsibility as dependent variables. Condition (LA/LE) was indicated as between-subject factor.

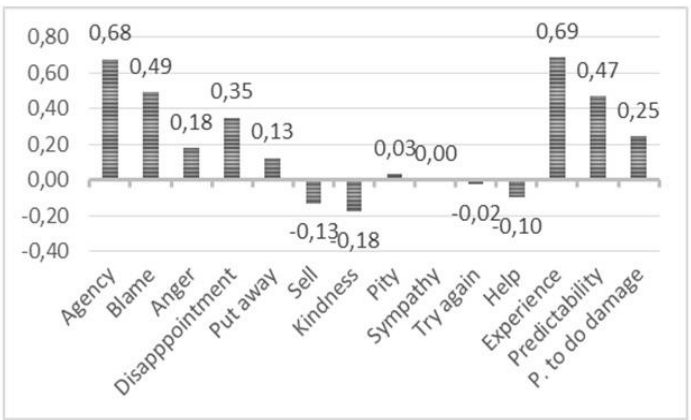

Fig. 2: Difference scores of means within the lack of ability and lack of effort (LA - LE) conditions.

\section{Results}

According to what was expected, participants attributed more agency to a NAO robot after seeing videos in which it displayed lack of effort $(M=2.80, S D=0.82)$ compared to videos in which it displayed lack of ability $(M=2.12$, $S D=0.61)$. Univariate tests expressed significant and large

\footnotetext{
1 Videos and complete survey can be found online: https://www.youtube.com/playlist?list=PLSQsUzV48QtG_YPY6kVcgC M8-YOcNqja; https://eu.qualtrics.com/jfe/preview/SV_6y4TuTii0CFnpch ${ }^{2}$ In this abstract we chose to focus on our main questions only. Therefore, additional analyses and results regarding these variables will not be discussed.
}

effects for the composite scores of agency $(F(1,61)=$ $13.601, p=.000$, eta $\left.{ }^{2}=.182\right)$, experience $(F(1,61)=$ $12.235, p=.001$, eta $\left.^{2}=.168\right)$, and predictability $(F(1,61)=$ $14.040, p=.000$, eta $\left.^{2}=.187\right)$. The results for the items of responsibility were mixed. While univariate tests for blame and disappointment revealed significant, medium effects (respectively: $F(1,61)=5.757, p=.019$, eta $^{2}=.086 ; F(1$, 61) $=9.704, p=.003, e t a^{2}=.137$ ), effects for the items anger, put away, sell, kindness, pity, sympathy, help and try again were not significant.

\section{Conclusion}

Similar to findings related to human interaction, the results of our study reveal that, in case of robots displaying behaviour that can be interpreted as lack of effort, humans tend to explain robotic behaviour by attributing agency. In case of failure, a robot displaying lack of effort - essentially refraining from 'trying' - may lead to blame and disappointment. However, it does not necessarily lead to negative affective and behavioural reactions such as anger, or wanting to shut the robot off and put it away. Results like these emphasize that we should be aware of potential diffusion of human responsibility when (advanced) robots create the impression that they are agents in the sense of actually controlling and intending their own actions. Our results also suggest that -in case of NAO robots- failure, or even reluctance for doing tasks is received well, illustrating a promisingly positive view on robots.

\section{References}

Asaro, P. 2013. A body to kick, but still no soul to damn: Legal perspectives on robotics. In Robot Ethics: The Ethical and Social Implications of Robotics, ed. Lin, K. Abney, and G. Bekey, 169186. Cambridge: MIT Press.

Clarke, R. 1994. Asimov's Laws for Robotics: Implications for Information Technology. Parts 1 and 2. Computer, part 1: 26(12): 53-61, 1993 and part 2: 27(1): 57-65), 1994.

Coleman, K.W. 2004. Computing and Moral Responsibility. In The Stanford Encyclopedia of Philosophy (Fall 2006 Edition), ed. Zalta, E.N. Stanford: The Metaphysics Research Lab.

Duffy, B.R. 2003. Anthropomorphism and the social robot. Robotics and Autonomous Systems, 42(3-4): 177-190.

Kim, T.; and Hinds, P. 2006. Who should I blame? Effects of autonomy and transparency on attributions in human-robot interaction. In Proceedings of ROMAN 06: The 15th IEEE International Symposium on Robot and Human Interactive Communication, 80-85. IEEE.

Koay, K.L.; Syrdal, D.S.; Walters, M.L.; and Dautenhahn. K. 2009. Five weeks in the robot house - exploratory human-robot interaction trials in a domestic setting. In Proceedings of the 2009 Second International Conferences on Advances in ComputerHuman Interactions, 219-226. IEEE. 
Malle, B.F.; Scheutz, M.; Arnold, T.; Voiklis, J.; and Cusimano, C. 2015. Sacrifice One for the Good of Many? People Apply Different Moral Norms to Human and Robot Agents. In Proceedings of the Tenth Annual ACM/IEEE International Conference on Human-Robot Interaction, 117-124. New York: ACM.

Malle, B.F.; Scheutz, M.; Forlizzi, J.; and Voiklis, J.; 2016.

Which Robot Am I Thinking about? The Impact of Action and Appearance on People's Evaluations of a Moral Robot. In The Eleventh Annual Meeting of the IEEE Conference on HumanRobot Interaction, 125-132. IEEE.

Moon, Y.; and Nass, C. 1998. Are computers scapegoats? Attributions of responsibility in human computer interaction. International Journal of Human-Computer Interaction, 49(1): 79-94.

Rudolph, U.; Roesch, S.C.; Greitemeyer, T.; and Weiner. B. 2004. A meta-analytic review of help giving and aggression from an attributional perspective. Cognition and Emotion, 18(6): 815848.

Serenko, A. 2007. Are interface agents scapegoats? Attributions of responsibility in human-agent interaction. Interacting With Computers, 19(2): 293-303.

Singer, P.W. 2011. Military robotics and ethics: A world of killer apps. Nature, 477(7365): 399-401.

Vilaza, G.N.; Haselager, W.F.G.; Campos, A.M.C; and Vuurpijl, L. 2014. Using games to investigate sense of agency and attribution of responsibility. In Proceedings of the 2014 SBGames, 393-399.

Weiner. B. 1995. Judgments of responsibility: A foundation for a theory of social conduct. New York/London: Guilford Press.

You, S.; Nie, J.; Suh, K.; and Sundar, S. 2011. When the robot criticizes you: Self-serving bias in human-robot interaction. In Proceedings of the 6th International Conference on Human Robot Interaction, 295-296. IEEE.

Złotowski, J.; Strasser, E.; and Bartneck, C. 2014. Dimensions of anthropomorphism: from humanness to humanlikeness. In Proceedings of the 2014 ACM/IEEE international conference on human-robot interaction, 66-73. New York: ACM. 